\title{
Towards Controlling Bucket Fill Factor in Robotic Excavation by Learning Admittance Control Setpoints
}

\author{
Heshan A. Fernando, Joshua A. Marshall, Håkan Almqvist and Johan Larsson
}

\begin{abstract}
This paper investigates the extension of an admittance control scheme toward learning and adaptation of its setpoints to achieve controllable bucket fill factor for robotic excavation of fragmented rock. A previously developed Dig Admittance Controller (DAC) is deployed on a 14-tonne capacity robotic load-haul-dump (LHD) machine, and full-scale excavation experiments are conducted with a rock pile at an underground mine to determine how varying DAC setpoints affect bucket fill factor. Results show that increasing the throttle setpoint increases the bucket fill factor and increasing the bucket's reference velocity setpoint decreases the bucket fill factor. Further, the bucket fill factor is consistent for different setpoint values. Based on these findings, a learning framework is postulated to learn DAC setpoint values for a desired bucket fill factor over successive excavation iterations. Practical implementation problems such as bucket stall and wheel-slip are also addressed, and improvements to the DAC design are suggested to mitigate these problems.
\end{abstract}

\section{Introduction}

In underground mining, articulated wheel loaders known as load-haul-dump (LHD) machines are utilized to excavate blasted rock from muck piles (i.e., piles of blasted rock, sometimes mixed with water and fine particles) at draw-points and haul this material to dump locations. Automating LHDs can improve safety and productivity of underground mining operations by removing operators from these hazardous and repetitive tasks. State-of-the-art technologies now offer autonomous tramming and

Heshan A. Fernando and Joshua A. Marshall

Mining Systems Laboratory, Queen's University, 25 Union St, Kingston, ON K7L 3N6 Canada, e-mail: \{heshan.fernando, joshua.marshall\}@queensu.ca

Håkan Almqvist and Johan Larsson

Rocktec Automation Division of Atlas Copco Rock Drills AB, Klerkgatan 21, SE-701 91, Örebro, Sweden e-mail: \{hakan.almqvist, johan.larsson\}@se.atlascopco.com 
Fig. 1 A boom and bucket manipulator commonly found on mobile wheel loaders. The boom link and bucket end-effector are actuated by hydraulic cylinders. The Dig Admittance Controller (DAC) requires measurement of the bucket cylinder position $\left(x_{a}\right)$ and measurement of the interaction force $\left(f_{r}\right)$ at the boom cylinders.

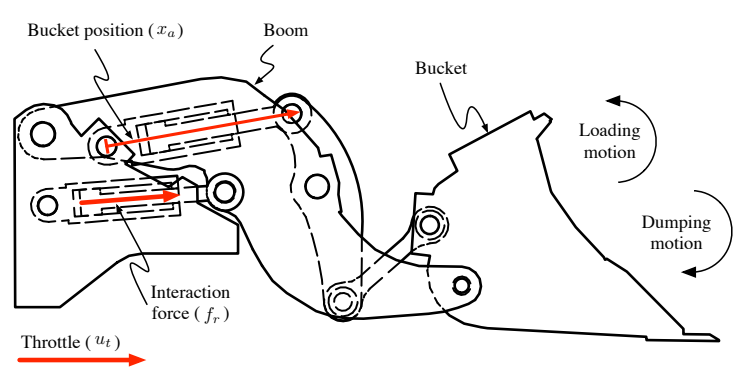

dumping for LHDs, but commercially viable technologies for robotic excavation (i.e., loading) of fragmented rock are still in early stages of development.

The main challenge in developing a successful robotic system for excavating fragmented rock is designing a controller that can effectively regulate the motion of an excavator bucket (e.g., Fig. 1) to dig through a rock pile and fill the bucket with material to a desired level at each excavation iteration (i.e., does not over-fill or under-fill). For excavating homogeneous media such as a sand and gravel piles, it is possible to use stereo-vision cameras or laser scanners to first scan the pile profile, plan a path trajectory to excavate a volume of material equivalent to the excavator's bucket, and apply robot motion control strategies to track the path with the bucket (Hemami and Hassani, 2009). However, pure motion control strategies are ineffective when excavating fragmented rock because subsurface obstacles can cause large position errors that result in saturated actuation (Maeda et al, 2015; Dadhich et al, 2016a). Further, cameras and laser scanners are not practical to implement in dark and dusty underground mining environments.

We believe that the path to successful excavation of fragmented rock is through "feel", rather than through "sight". Specifically, we believe that feedback of measured bucket-rock interaction forces to an admittance control scheme is effective for regulating an excavator's bucket motion to dig through a rock pile and fill the bucket with material (Marshall et al, 2008). Further, we hypothesize that the bucket fill factor (or payload weight) can be controlled by varying the setpoint parameters of an admittance control scheme for robotic excavation of fragmented rock. In this paper, we present results of full-scale experiments with a 14-tonne capacity LHD machine and an underground muck pile that support our hypothesis.

\subsection{Related Work}

Admittance control for robotic excavation of fragmented rock was initially postulated by Marshall et al (2008) based on results of full-scale excavation experiments with expert operators and an instrumented wheel loader. Analysis of the force and motion data at the loader's bucket hydraulic cylinder showed that during excava- 
tion, operators controlled the bucket motion in an attempt to maintain a level of interaction force-though the operators were not conscious of this underlying fact. This led Marshall et al (2008) to postulate an admittance control scheme for robotic excavation of fragmented rock that regulates bucket motion based on feedback of interaction forces. Compared to intelligence-based approaches to robotic excavation (Dadhich et al, 2016b) that require substantial training data and exhibit unpredictable behaviour in untrained situations, the admittance control scheme proposed by Marshall et al (2008) provides an effective framework for use in practical implementations.

Recent research work (Dobson et al, 2015, 2017) has developed a Dig Admittance Controller (DAC) for robotic excavation based on the admittance control scheme proposed by Marshall et al (2008). The DAC was tested in full-scale robotic excavation experiments, which showed that a 14-tonne capacity LHD that was robotically controlled using the DAC excavated larger payloads of fragmented rock, more consistently, compared to an expert operator excavating the same rock pile using the same machine (Dobson et al, 2015). However, a challenge in implementing the DAC was selecting the proper control parameters for a desired bucket fill factor; parameter tuning was unintuitive due to lack of an explicit model for bucket-rock interaction. Manually tuning the DAC parameters worked well for a rock pile with particular characteristics (e.g., bulk density, fragmentation size and cohesion), but the control parameters required re-tuning to excavate a desired bucket fill factor from a pile with different characteristics.

In practice, pile characteristics are difficult to model explicitly, and these characteristics can evolve throughout the life of an excavation operation. Thus, for practical implementation in robotic excavation, the DAC requires extension by learning and adaptation so that optimal parameters for a desired bucket fill factor are automatically learned and adapted at each excavation iteration.

Research in learning-based interaction control has developed in recent years due to increased interest in emerging field and service robot applications such as human-robot collaboration (Haddadin and Croft, 2016) and robot-assisted surgery (Kim et al, 2016). A fundamental requirement for robots in these applications is to maintain desired performance of a particular task while physically interacting with unstructured and evolving environments. Due to their robustness and stability in compliant manipulation tasks, interaction controllers based on the impedance and admittance control paradigms (Villani and De Schutter, 2016) are often chosen for extension by learning and adaptation in these emerging applications.

Much of the research for learning-based interaction control has focused on extension of impedance controllers toward adaptive controllers through learning of impedance parameters or desired trajectories (Haddadin and Croft, 2016). Many of these "variable impedance" control approaches are based on iterative learning strategies where the objective is to learn the optimal impedance/admittance parameters through successive interaction with the environment in repetitive tasks. Applications have mainly focused on human-robot collaboration (Yamawaki et al, 2016; Wang et al, 2016; Dimeas and Aspragathos, 2015; Li and Ge, 2014; Buchli et al, 2011). Typically, a cost function is defined using interaction force and/or trajectory 
tracking error. Impedance parameters, such as a virtual damping coefficient, are then updated over a trajectory (i.e., gain scheduling) by minimizing the cost function. Stability of these methods have been analyzed by Kronander and Billard (2016), and it was found that varying impedance control parameters can lead to instability. Impedance control with constant gains are typically stable when interacting with passive environments, but instability can occur when the gains are varying throughout the task.

\subsection{About this Paper}

In this paper, through full-scale field experiments, we investigate learning and adaptation of Dig Admittance Control (DAC) setpoints in order to achieve controllable bucket fill factor in robotic excavation of unstructured and evolving media, such as fragmented rock. First, the DAC scheme is presented in Section 2. Details of the LHD, muck pile and experiment procedure are provided in Section 3. Experiment results for varying DAC setpoints on bucket fill factor are presented and analyzed in Section 4. These results are further discussed in Section 5 in order to suggest improvements, and to postulate a learning framework for adapting the DAC setpoints. Finally, future directions and upcoming experimental work for continuation of this research are presented in Section 6.

\section{Approach to Robotic Excavation: The Dig Admittance Controller}

A block diagram of the Dig Admittance Controller (DAC), which was postulated by Marshall et al (2008) and tested by Dobson et al (2017) for robotic excavation of fragmented rock using wheel loaders, is shown in Figure 2. In this scheme, the admittance controller $Y(s)$ attempts to comply the wheel loader's bucket motion with the rock pile interaction and react to measured interaction forces by modifying the reference motion trajectory $x_{c}$ to a position-controlled bucket actuator (i.e., hydraulic cylinder). Thus, in a discrete-time implementation with a constant period $T$ and a proportional admittance $Y(s)=K_{A}$ (as used by Dobson et al (2017)), the reference motion command to the bucket hydraulic cylinder at a time-step $k$ becomes

$$
x_{c, k}=x_{c, k-1}+T\left(v_{f, k}+v_{d}\right),
$$

where $v_{d}$, is a constant nominal velocity setpoint for the bucket curl rate, and

$$
v_{f, k}=K_{A}\left(f_{d}-\hat{f}_{r, k}\right)
$$


is a computed velocity change that is proportional to the error between a constant target force setpoint $f_{d}$ and the measured interaction force $\hat{f}_{r, k}$. The interaction force $\hat{f}_{r, k}$ is calculated from pressure measurements at the boom cylinders as the boom is not actuated during excavation. Thus, the boom pressures measure only interaction forces and are not contaminated by other forces due to input signals. A constant forward throttle percent $u_{t}$ is applied to the loader during excavation in order for the bucket to maintain contact with the pile.

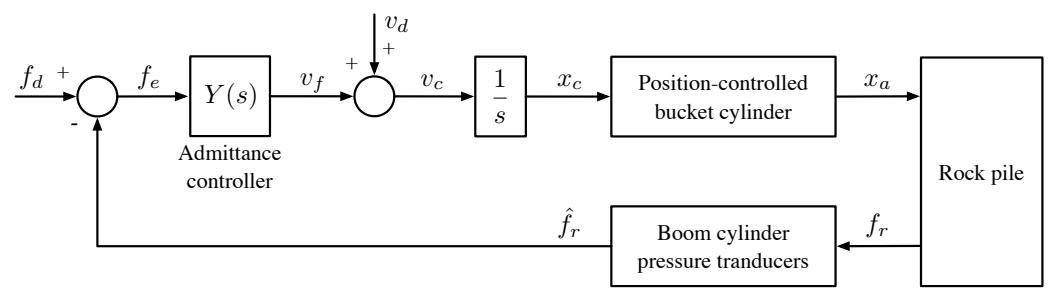

Fig. 2 A block diagram of the Dig Admittance Controller (DAC) for robotic excavation of fragmented rock postulated by Marshall et al (2008) and tested by Dobson et al (2017) in full-scale experiments

The challenge in implementing the DAC in practice is determining how to best select control setpoints $f_{d}$ and $v_{d}$, and throttle setpoint $u_{t}$ to achieve desired bucket fill factor (typically measured as payload in tonnes) at each excavation iteration. The values of these parameters are dependent on the physical characteristics of the excavation media (e.g., bulk density, fragmentation and cohesion), which are difficult to model prior to excavation and typically evolve throughout the life of the excavation operation.

In their experiments, Dobson et al (2017) manually tuned the setpoints until a desired bucket fill factor was achieved. Once tuned, the DAC performed well in excavating consistent payloads from a particular rock pile, but the parameters required retuning when the pile characteristics changed. In our investigation, we are interested in determining the effects of varying the DAC setpoints on the bucket fill factor. If trends can be observed in the fill factor for varying setpoint values, then a learning framework can be developed to automatically learn and adapt the parameters for a desired fill factor over successive excavation iterations.

\section{Experiment Apparatus and Methodology}

Full-scale robotic excavation experiments described in this paper were conducted using an automation-ready Atlas Copco ST14 load-haul-dump (LHD) machine and a muck pile located at an underground mining test-facility at Kvarntorp, Sweden. Figure 3 shows images of the ST14 and muck pile. 


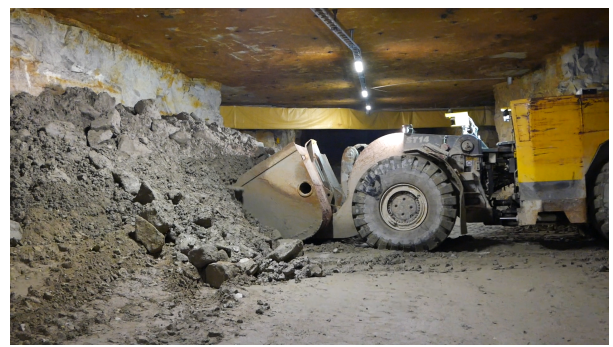

(a) ST14 LHD and muck pile

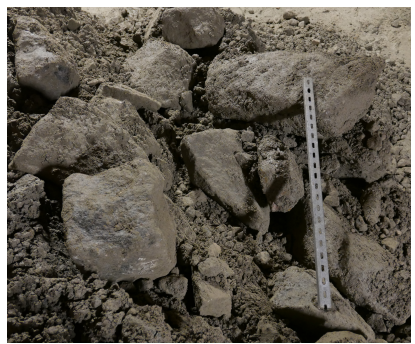

(b) Sample of rocks in muck pile

Fig. 3 Images of the ST14 LHD machine and muck pile used for experiments described in this paper. A one metre long rod is placed on a sample of rocks as a length reference.

\subsection{ST14 LHD Specifications}

The Atlas Copco ST14 LHD machine is an articulated, low-profile, wheel loader that is designed for loading, hauling and dumping blasted rock in underground mining operations. This LHD has a 14-tonne payload capacity and a loading mechanism with a boom and bucket linkage as shown in Figure 1. Two parallel hydraulic cylinders actuate the boom, and a single hydraulic cylinder, connected via a Z-bar linkage, actuates the bucket. As depicted in Figure 1, bucket cylinder extension corresponds to bucket curling motion and bucket cylinder retraction corresponds to bucket dumping motion. The ST14 transmission is powered by a 335 HP diesel engine, and the hydraulic actuators (i.e., for boom, bucket and steering) are powered by two load-sensing variable displacement pumps. The hydraulic system is designed in a closed-centre configuration, so all hydraulic actuators receive maximum power from the variable displacement pumps. Electrohydraulic servo valves control flow to the hydraulic cylinders for actuation.

\subsection{Sensors and Data Acquisition}

Automation-ready ST14 LHD machines are equipped with programmable control systems and sensors that provide information about the machine's status. These sensors include pressure transducers that measure the boom cylinders' base and rod-side hydraulic pressures, a linear position transducer that measures the bucket cylinder extension length, an absolute encoder that measures the boom angle, and a cogwheel that measures the transmission speed. Thus, no additional hardware was required to implement the admittance controller on the ST14.

The ST14 control system operates in real-time at a frequency of $20 \mathrm{~Hz}$ for reading sensor measurements, sending actuator commands and logging data. This control frequency is adequate for implementing the admittance controller because bucketrock interaction dynamics typically have very low bandwidth (Maeda et al, 2015). 
To conduct the robotic excavation experiments, the admittance control scheme described in Figure 2 was programmed onto the ST14's control system. The interaction force $\hat{f}_{r}$ was calculated from hydraulic pressure measurements at the base and rod sides of the boom cylinders as $\hat{f}_{r}=P_{1} A_{1}-P_{2} A_{2}$ where $P_{1}$ and $A_{1}$ are the boom cylinders' base pressure and cross-sectional area, respectively, and $P_{2}$ and $A_{2}$ are the boom cylinders' rod-side pressure and cross-sectional area, respectively.

Actuator commands to the bucket cylinder's servo valve are sent as digital signals that correspond to the valve's spool displacement. These command signals are normalized between -1 and +1 , where negative values correspond to cylinder retraction and positive commands correspond to cylinder extension. Valve dead-bands are reduced in the control software so that the valve commands correspond to a nearlinear response from the cylinder.

Payload weight $W$ is calculated using a static load analysis with the boom raised to a specified angle. Boom cylinder pressure measurements are used to obtain the forces required for the analysis. We were unable to rigorously test the accuracy of the load weighing system due to lack of a calibration weight, but the system's precision was determined by raising and lowering the boom five times with a filled bucket. The largest absolute deviation (i.e., the largest difference between a measurement and the sample mean) was determined to be 0.2 tonnes.

\subsection{Excavation Media}

A muck pile, shown in Figure 3, was used for all robotic excavation trials presented in this paper. The back of the pile was confined to a wall in a test area of an underground mine at Kvarntorp, Sweden. This muck pile consisted of a mixture of clay, fine gravel and large fragments of blasted rock ( 30 to $70 \mathrm{~cm}$ in nominal diameter), which is representative of a real muck pile found in underground mining. However, real muck piles are typically larger and more confined with a continuous flow of material from a blasted stope above the muck pile.

\subsection{Robotic Excavation Trials Procedure}

Robotic excavation trials followed a procedure of manually positioning the loader in front of the pile, executing the excavation algorithm, manually backing out of the pile, weighing the excavated payload and dumping the excavated material back on to the pile. At the start of each excavation trial, the admittance controller's throttle parameters $u_{t}$ and reference velocity setpoint $v_{d}$ were set in the ST14 control system, and the excavation algorithm was executed to automate the following steps:

1. Use position controllers to move boom and bucket to entry positions.

2. Apply throttle $u_{t}$ to drive the LHD forward into the pile and penetrate the pile with the bucket. 
3. When measured force $\hat{f}_{r}$ exceeds an entry force threshold, $f_{\text {entry }}$, execute the admittance controller to dig through the pile and fill the bucket.

4. When bucket is fully curled, deactivate the admittance controller and return throttle to zero.

The lack of overhead confinement on our muck pile resulted in material buildup at the tip of the bucket at the end of excavation. Thus, at the end of each dig, an operator manually shook the bucket to allow this material to fall back into the bucket prior to load weighing.

\section{Experiments and Analysis}

Robotic excavation experiments were conducted to determine the effects of changing the Dig Admittance Controller's (DAC) target force setpoint $f_{d}$, throttle setpoint $u_{t}$ and reference velocity setpoint $v_{d}$ on excavated payloads $W$. Results and analysis from these experiments are presented in the following subsections.

\subsection{Preliminary Experiments}

In preliminary robotic excavation experiments that varied the constant target force setpoint $f_{d}$, it was determined that setting a low $f_{d}$ can lead to bucket stall during excavation. Measured force and motion data from a stalled trial is shown in Figure 4. It can be seen that as the bucket curls, the measured interaction force $\hat{f}_{r}$ continues to increase. Eventually, $\hat{f}_{r}$ increases beyond the target force $f_{d}$, causing the velocity change $v_{f}$ to negate the reference velocity $v_{d}$ to the bucket. This effectively stalls bucket motion and prevents the LHD from moving into the pile.

\subsubsection{Target Force Adaptation}

To prevent bucket stall situations from degrading the performance of a setpoint learning framework, we implemented an averaging filter to adapt the target force setpoint $f_{d}$ to be a moving average of the measured interaction force

$$
\bar{f}_{d, k}=\bar{f}_{d, k-1} \frac{n-1}{n}+\frac{\hat{f}_{r, k}}{n},
$$

where $\bar{f}_{d, k}$ is the new average target force setpoint at the current timestep $k, \bar{f}_{d, k-1}$ is the average target sepoint from the previous timestep, $n$ is the averaging window size, and $\hat{f}_{r, k}$ is the measured interaction force at the current time step. This averaging algorithm is computationally more efficient than calculating the true moving average. 


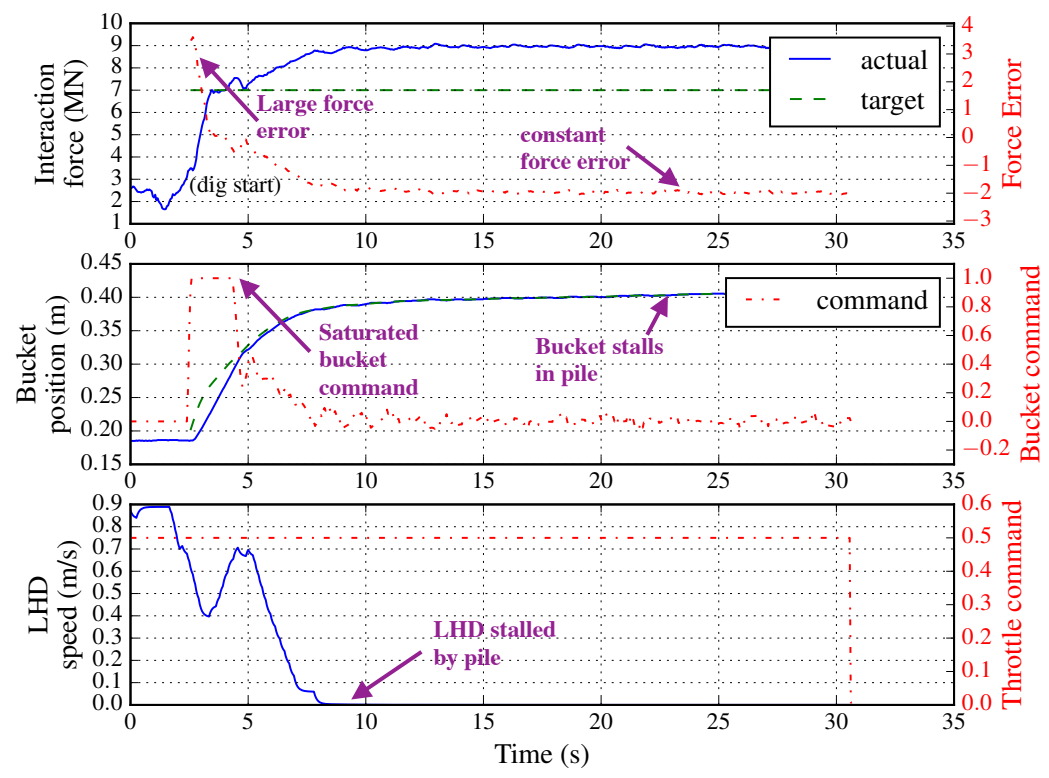

Fig. 4 Force and motion data from excavation trials with a constant target force setpoint $f_{d}=7.0$ $\mathrm{MN}$, reference velocity setpoint $v_{d}=0.040 \mathrm{~m} / \mathrm{s}$ and throttle setpoint $u_{t}=50 \%$. Setting a low, constant, $f_{d}$ can result in bucket stall during excavation.

The target force adaptation scheme proposed above is based on analysis of data from 25 robotic excavation trials with different, constant, $f_{d}$ values. Analysis of the force signals from these consistently showed that the measured interaction forces always increase as the bucket curls, but the magnitude and profile of the force signal is dependent on many factors (e.g., material accumulated in the bucket, contact with the pile, throttle, etc.), so it is difficult to predetermine a suitable profile for the target force setpoint at each excavation iteration.

Ideally, the target force should follow the nominal level of interaction forces, so that changes in the interaction forces beyond this level correspond to appropriate velocity changes $v_{f}$ by the admittance controller. We believe that the moving average technique achieves this, although a lag is present due to averaging. Preliminary testing found that $n=20$ provides a suitable averaging window size.

\subsection{Robotic Excavation Trials for Varying $u_{t}$ and $v_{d}$}

The excavation experiments were conducted by deploying the DAC, described in Section 2 (with the target force setpoint adaptation modification described in Section 4.1.1), on the ST14 LHD machine, and following the excavation procedure outlined in Section 3.4. The experiment variables are the DAC throttle setpoint $u_{t}$ and reference velocity setpoint $v_{d}$. All other control parameters: admittance con- 
troller gain $K_{A}$, pre-entry boom and bucket positions, entry force threshold $f_{\text {entry }}$, and target force setpoint moving average window size $n$ were manually tuned at the start and kept constant throughout the trials.

The uncontrollable variables in these trials are pile shape and pile consistency, which introduce some variation in the payload results. Because the muck pile used in these experiments did not have a continuous flow of material, the excavated payloads were dumped back onto the pile after each excavation trial. The pile shape was adjusted twice during the trials as a lack of confinement at the sides caused the pile to push out and decrease in depth. We are not concerned with pile changes between trials in this experiment as the DAC should be robust to pile variations.

The experiment matrix for the excavation trials is given in Table 1. Combinations of four different $v_{d}$ values and three different $u_{t}$ values were tested. A $v_{d}$ value of $0.100 \mathrm{~m} / \mathrm{s}$ was tested because this results in saturation of the bucket command during digging; these trials provide reference payloads for simply curling the bucket at maximum speed after pile penetration. The initial plan was to conduct four trials for each parameter combination; however, significant wheel slip at $70 \%$ throttle setpoint created deep trenches in roadway at the tip of the muck pile. Wheel slip can also cause the LHD's tires to heat and possibly burst, so we did not pursue many excavation trials at high throttle values. In the end, we were able to conduct five excavation trials at $u_{t}=70 \%$, which gave 37 successful trials for results and analysis. All trials were conducted in a random order to avoid pile variations from biasing payload results.

Table 1 Experiment matrix showing the number of excavation trials completed for different combinations of throttle setpoint $u_{t}$ and reference velocity setpoint $v_{d}$ in the DAC.

\begin{tabular}{|l|c|c|c|}
\hline$v_{d} u_{t}$ & $30 \%$ & $50 \%$ & $70 \%$ \\
\hline $0.020 \mathrm{~m} / \mathrm{s}$ & 4 & 4 & 0 \\
\hline $0.040 \mathrm{~m} / \mathrm{s}$ & 4 & 4 & 1 \\
\hline $0.060 \mathrm{~m} / \mathrm{s}$ & 4 & 4 & 2 \\
\hline $0.100 \mathrm{~m} / \mathrm{s}$ & 4 & 4 & 2 \\
\hline
\end{tabular}

\subsubsection{Results and Analysis}

The DAC, modified with target force setpoint adaptation, worked well in our robotic excavation experiments-there were no bucket stall problems. Thus, the ST14 LHD was able to autonomously excavate the muck pile and load the bucket in all excavation trials. Trials with $u_{t}=70 \%$ were deactivated when significant wheel slip was observed; however, we expect that these trials would also have had successful excavation results if wheel slip had not occurred. The issue of wheel slip is addressed after presenting the payload results for the successful excavation trials below.

Figure 5 shows plots of average payloads from robotic excavation trials with different DAC setpoints, $u_{t}$ and $v_{d}$. This plot shows that increasing $u_{t}$ increases pay- 
load, and increasing $v_{d}$ decreases payload. Despite pile variations, the excavated payloads for each parameter combination are consistent. These results affirm our hypothesis that controllable bucket fill factor can be achieved by controlling the admittance of the bucket rock interaction. For the trial sets with $u_{t}=30 \%$ and $u_{t}=50$ $\%$, the maximum standard deviation for payloads from was 0.6 tonnes (recall that the load weighing system's precision is 0.2 tonnes). Thus, the minimum controllability of bucket payloads that we would be able to achieve with this robotic excavation system is approximately 0.6 tonnes.

Fig. 5 Payload results from robotic excavation trials for varying throttle parameter $u_{t}$ and reference velocity $v_{d}$ in the Dig Admittance Controller. Data points show the average values from all trials for a each parameter combination (see Table 1 for trial information); error bars indicate the standard deviations.

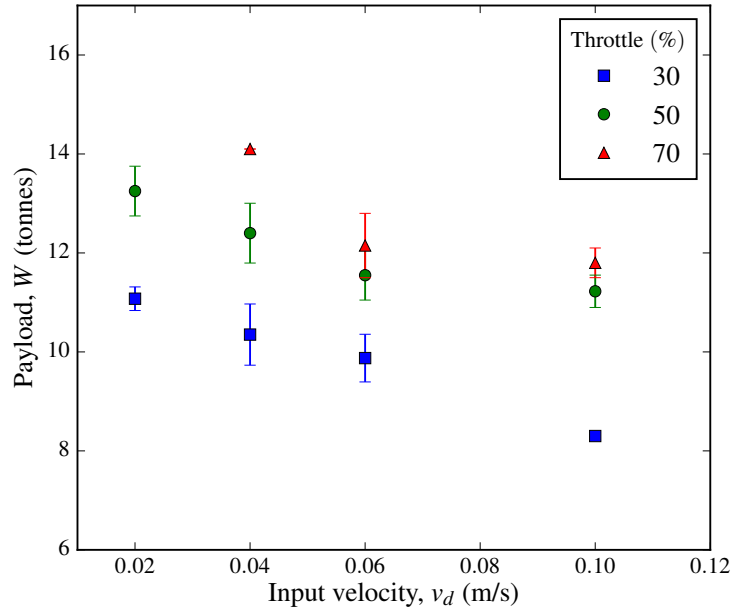

Wheel slip was observed for trials with low $v_{d}$ and high $u_{t}$. Based on observations during the excavation trials, we believe that this is due to slow bucket curl rate at the start of the dig. Slow bucket curl rate suggests low admittance in the control scheme, which means that the controller is not reacting quickly enough to increasing forces. Figure 6 shows measured force and motion data from a trial with $u_{t}=50 \%$ and $v_{d}=0.020 \mathrm{~m} / \mathrm{s}$. The force data shows that the moving average force target lags behind the measured force when forces are increasing. Because the force error is always negative, the velocity change $v_{f}$ acts to reduce the reference velocity $v_{c}$, resulting in slow curl rate at the start of the dig cycle. Slow bucket curl rate causes the LHD to become stalled by the pile, which results in small amounts of wheel slip. Analysis of the force error signal indicates sharp increases in forces at the start of the dig, which should correspond to increases in bucket curl rate to admit the LHD into the pile. We believe that introducing a derivative control action to the admittance controller could address this issue. 


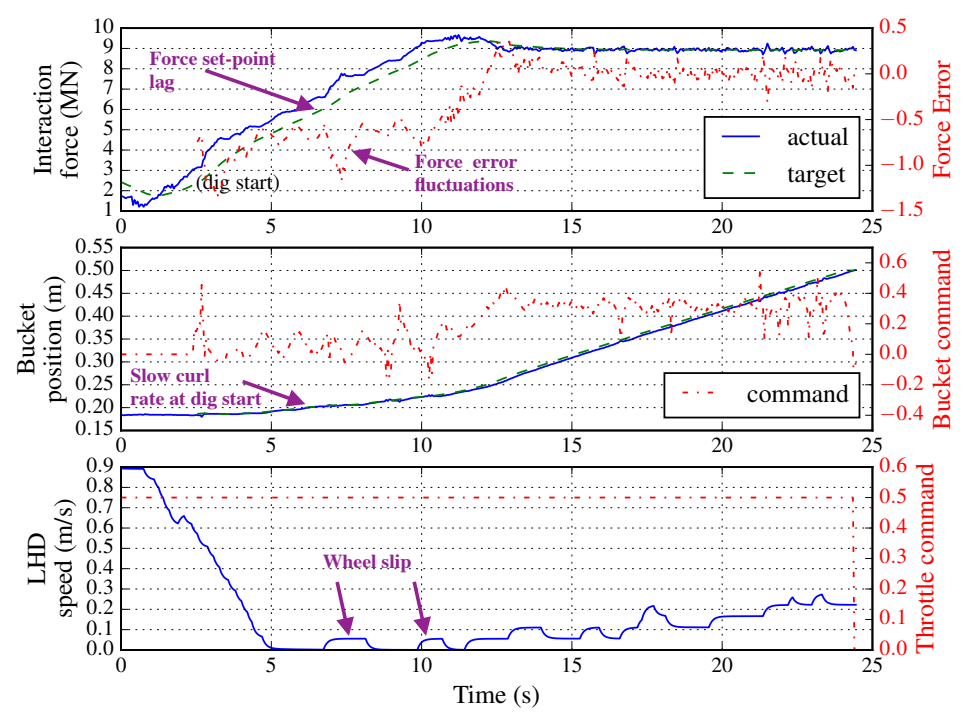

Fig. 6 Force and motion data from a robotic excavation trial using the Dig Admittance Controller modified with the moving average target force setpoint $\left(\bar{f}_{d}\right)$. This data is from a trial with throttle setpoint $u_{t}=50 \%$ and reference velocity setpoint $v_{d}=0.020 \mathrm{~m} / \mathrm{s}$.

\section{Setpoint Learning for Controllable Bucket Fill: Discussion}

Results from robotic excavation trials suggest that controlling the admittance of the excavation process by changing the throttle setpoint $u_{t}$ and reference velocity setpoint $v_{d}$ allow controllable bucket fill. Based on these results and subsequent analysis of data, the following discussion proposes improvements to the admittance controller design and postulates a learning framework for adapting these two setpoints over excavation iterations.

The moving average target force setpoint $\bar{f}_{d}$ modification to the Dig Admittance Controller (DAC) worked well in eliminating bucket stall situations during excavation; however, lag in target-force adaptation results in slow bucket curl at the dig start, which leads to wheel slip. Increasing the admittance gain $K_{A}$ would increase the response of the velocity change $v_{f}$ resulting in oscillatory behaviour for the bucket, which is not ideal. Analysis of the force error signals from excavation trials suggest that adding a derivative control action to the admittance controller as $Y(s)=K_{A} f_{e}+B_{A} \dot{f}_{e}$ might improve the reaction of the velocity change for greater rates of change in the interaction forces. Consideration will need to be given to filter the significant amount of noise present in the force measurement signal prior to calculating its derivative. 
There remains the question of how to best learn $u_{t}$ and $v_{d}$ to control bucket fillfactor. Both setpoints contribute to bucket payload, so it might be possible to learn both by using a simple gradient following algorithm at each excavation iteration, $j$ :

$$
\begin{aligned}
& u_{t, j+1}=u_{t, j}+\alpha\left(W_{d}-W_{j}\right) \\
& v_{d, j+1}=v_{d, j}-\beta\left(W_{d}-W_{j}\right),
\end{aligned}
$$

where $W_{d}$ is the desired bucket fill factor (or payload) and $\alpha, \beta>0$ are learning rates that must be tuned. Updating both setpoints at each excavation iteration may cause oscillations in the learning, so it might be better to give a fixed value to one setpoint and learn the remaining setpoint as per above learning laws. More excavation experiments are required to test these hypotheses.

\section{Conclusions and Future Work}

Results of full-scale robotic excavation experiments with a 14-tonne capacity loadhaul-dump vehicle and an underground muck pile show that varying the throttle and reference velocity setpoints of a previously developed Dig Admittance Controller (DAC) for robotic excavation of fragmented rock allows controllable bucket fill factor (i.e., payload). Based on these results, we postulate a learning algorithm that learns the DAC throttle and velocity setpoints based on a performance metric of payload error at each excavation iteration. Further, we suggest improvements to the DAC for adapting the target force set-point and introducing derivative action in the control law for better reaction to large increases in measured interaction forces. Future work proposes to test the proposed learning scheme with full-scale excavation experiments on different types of excavation media such as a gravel pile and a fragmented rock pile.

Acknowledgements This work was supported in part by the Natural Sciences and Engineering Research Council of Canada (NSERC) under project RGPIN-2015-04025, the Swedish Knowledge Foundation (KK-stiftelsen) under project 20150282, and by Atlas Copco Rock Drills AB (Sweden). This work was completed while the second author was a Visiting Professor at the Centre for Applied Autonomous Sensor Systems (AASS) in the School of Science and Technology at Örebro University, Sweden.

\section{References}

Buchli J, Stulip F, Theodorou E, Schaal S (2011) Learning variable impedance control. The International Journal of Robotics Research 30(7):820-833

Dadhich S, Bodin U, Andersson U (2016a) Key challenges in automation of earthmoving machines. Automation in Construction 68:212-222 
Dadhich S, Bodin U, Sandin F, Andersson U (2016b) Machine learning approach to automatic bucket fill. In: Proceedings of 24th Mediterranean Conference on Control and Automation, Athens, Greece, pp 1260-1265

Dimeas F, Aspragathos N (2015) Reinforcement learning of variable admittance control for human-robot co-manipulation. In: Proceedings of the 2015 IEEE/RSJ International Conference on Intelligent Robots and Systems (IROS), Hamburg, Germany, pp 1011-1016

Dobson AA, Marshall JA, Larsson J (2015) Admittance control for robotic loading: Underground field trials with an LHD. In: Proceedings of the 10th Conference on Field and Service Robotics (FSR), Toronto, Ontario, Canada

Dobson AA, Marshall JA, Larsson J (2017) Admittance control for robotic loading: Design and experiments with a 1-tonne loader and a 14-tonne LHD. Invited paper in the special issue on Field and Service Robotics in the Journal of Field Robotics 34(1):123-150

Haddadin S, Croft E (2016) Springer Handbook of Robotics, 2nd edn, Springer International Publishing, Switzerland, chap 69-Physical human-robot interaction, pp 1835-1874

Hemami A, Hassani F (2009) An overview of autonomous loading of bulk material. In: Proceedings of the 26th International Symposium on Automation Robotics in Construction (ISARC), Austin, Texas, pp 405-411

Kim YJ, Seo J, Kim H, Kim KG (2016) Impedance and admittance control for respiratorymotion compensation during robotic needle insertion - a preliminary test. The International Journal of Medical Robotic and Computer Assisted Surgery pp $1-10$

Kronander K, Billard A (2016) Stability considerations for variable impedance control. IEEE Transactions on Robotics 32(5):1298-1305

Li Y, Ge S (2014) Impedance learning for robots interacting with unknown envirnonments. IEEE Transactions on Control Systems Technology 22(4):14221432

Maeda GJ, Manchester IR, Rye DC (2015) Combined ILC and disturbance observer for the rejection of near-repetitive disturbances, with application to excavation. IEEE Transactions on Control Systems Technology 23(5):1754-1769

Marshall JA, Murphy PF, Daneshmend LK (2008) Toward autonomous excavation of fragmented rock: Full-scale experiments. IEEE Transactions on Automation Science and Engineering 5(3):562-566

Villani L, De Schutter L (2016) Springer Handbook of Robotics, 2nd edn, Springer International Publishing, Switzerland, chap 9-Force Control, pp 195-220

Wang C, Li Y, Ge SS, Lee TH (2016) Reference adaptation for robots in physical interactions with unknown environments, accepted for inclusion in future issue of IEEE Transactions on Cybernetics, 18 May 2016, DOI 10.1109/TCYB.2016.2562698

Yamawaki T, Ishikawa H, Yashima M (2016) Iterative learning of variable impedance control for human-robot cooperation. In: Proceedings of the 2016 IEEE/RSJ International Conference on Intelligent Robots and Systems (IROS), Daejeon, Korea, pp 839-844 